\title{
Observations of the gamma-ray emission from the Quiescent Sun with Fermi Large Area Telescope during the first 7 years in orbit
}

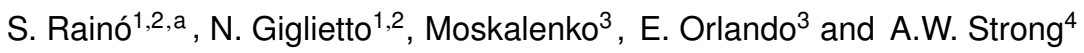 \\ ${ }^{1}$ Dipartimento Interateneo di Fisica "M.Merlin" dell'Universitá e del Politecnico di Bari, I-70126, Bari, Italy \\ ${ }^{2}$ Istituto Nazionale di Fisica Nucleare, Sezione di Bari, I-70126, Bari, Italy \\ ${ }^{3}$ W. W. Hansen Experimental Physics Laboratory, Kavli Institute for Particle Astrophysics and Cosmology, \\ Department of Physics and SLAC National Accelerator Laboratory, Stanford University, Stanford, California \\ 94305, USA \\ ${ }^{4}$ Max-Planck Institut für extraterrestrische Physik, 85748 Garching, Germany
}

\begin{abstract}
The high energy gamma-ray emission from the quiescent Sun is due to the interactions of cosmic ray (CR) protons and electrons with matter and photons in the solar environment. Such interactions lead to two component gamma-ray emission: a disk-like emission due to the nuclear interactions of CR protons and nuclei in the solar atmosphere and a space extended emission due to the inverse Compton (IC) scattering of CR electrons off solar photons in the whole heliosphere. The observation of these two solar emission components may give useful information about the evolution of the solar cycle by probing two different CR components (proton and electrons) in regions not directly accessible by direct observations. We present the results of the observations of the Sun with Fermi-LAT in the first 7 years on orbit, with the exception of the flaring periods. Significantly large photon statistics and improved processing performance with respect to previous analysis allow us to explore both components of the emission in greater details and perform better comparisons of data with current models of the IC component. This allows us to probe $\mathrm{CR}$ electrons in the inner heliosphere which is not possible by other methods. Moreover, the longer period of observations allows us to study the variations of the emission between the maximum and the minimum of the solar cycle.
\end{abstract}

\section{Introduction}

The high energy gamma-ray emission from the quiescent Sun is due to the interactions of cosmic ray (CR) protons and electrons with matter and photons in the solar environment. Such interactions lead to two component gamma-ray emission: a disk-like emission due to the nuclear interactions of CR protons and nuclei in the solar atmosphere and a space extended emission due to the inverse Compton (IC) scattering of CR electrons off solar photons in the whole heliosphere [1] [2] . While the IC emission is brightest in the region within a few degrees from the Sun, even at larger elongation angles it can be comparable in intensity to the isotropic (presumably extra-galactic) gamma-ray background

\footnotetext{
ae-mail: silvia.raino@ba.infn.it
} 

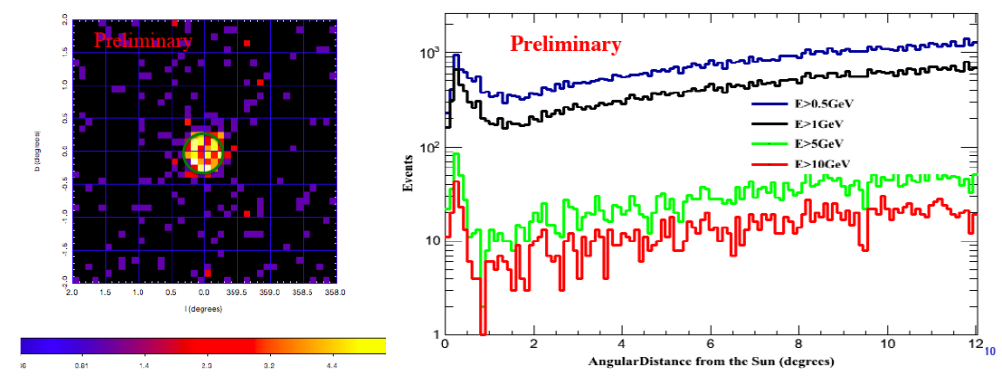

Figure 1. Left: Photons counts map of the solar emission components above $10 \mathrm{GeV}$. Right: Sun events as a function of the angular distance from the Sun, for different energy thresholds. From top to bottom: $\mathrm{E}_{\min }=0.5 \mathrm{GeV}$, $1 \mathrm{GeV}, 5 \mathrm{GeV}, 10 \mathrm{GeV}$.

[3]. The flux for both components of the CR-induced emission is expected to change over the solar cycle due to the change of the heliospheric flux of the Galactic CRs in anticorrelation with the variations of the solar activity. The observation of these two solar emission components may give useful information about the evolution of the solar cycle by probing two different CR components (proton and electrons) in regions not accessible by direct measurements.

\section{Data Selection and background}

In our analysis we have evaluated the gamma-ray flux from the Sun using the data collected by the Fermi LAT in the first 7.5 years of operation from 2008 August 4 up to 2015 December 31 . The data set used is much larger than the previously published [4], moreover the data are reprocessed with the new Pass 8 reconstruction and event-level analysis that is a comprehensive revision of the entire analysis chain and provides improved processing performance with respect to previous analysis: wider energy range, larger acceptance, better Point Spread Function (PSF), better background rejection, better control of systematic uncertainties [5]. We select events in an energy range between $100 \mathrm{MeV}$ and $30 \mathrm{GeV}$ in a region of interest (ROI) of $20^{\circ}$ centered on the Sun position. To reduce the contamination by the albedo gamma-ray emission coming from CR interactions in the Earth's upper atmosphere our selection is refined by selecting events with zenith angles $<100^{\circ}$. To reduce the systematic uncertainties due to bright gamma-ray emission from the Galactic plane we exclude the data taken when the Sun was within $30^{\circ}$ of the plane. We further excluded the periods when the Sun was within $20^{\circ}$ from the Moon or any other bright celestial source with integral flux $\mathrm{F} \geq 2 \times 10^{-7} \mathrm{~cm}^{-2} \mathrm{~s}^{-1}$ above $100 \mathrm{MeV}$, as reported in the 3FGL catalog [6]. Since solar flares gamma rays are known to be produced directly from nuclear interactions of accelerated protons and heavier ions we remove all flaring periods observed from the considered data set in order to focus on the quiet component of the sun emission. Because the Sun is a moving source, the analysis of its emission requires a special treatment, so the data are selected in a moving frame centered on the instantaneous solar position. Fig. 1 shows a Sun-centered counts map of the solar emission components above $10 \mathrm{GeV}$ and the Sun events as a function of the elongation angle for different energy thresholds (from top to bottom, $\mathrm{E}_{\text {min }}=0.5 \mathrm{GeV}, 1 \mathrm{GeV}, 5 \mathrm{GeV}, 10 \mathrm{GeV}$ ). The peak corresponds to the disk events in the centre of the reference frame ( $\theta=0$, where $\theta$ is the elongation angle). The correct evaluation of the background in the region around the Sun is of considerable importance for the analysis of the weak extended IC emission. The latter is expected to decrease with $\sim 1 / \theta$ [1]. The background is mainly due to the diffuse 

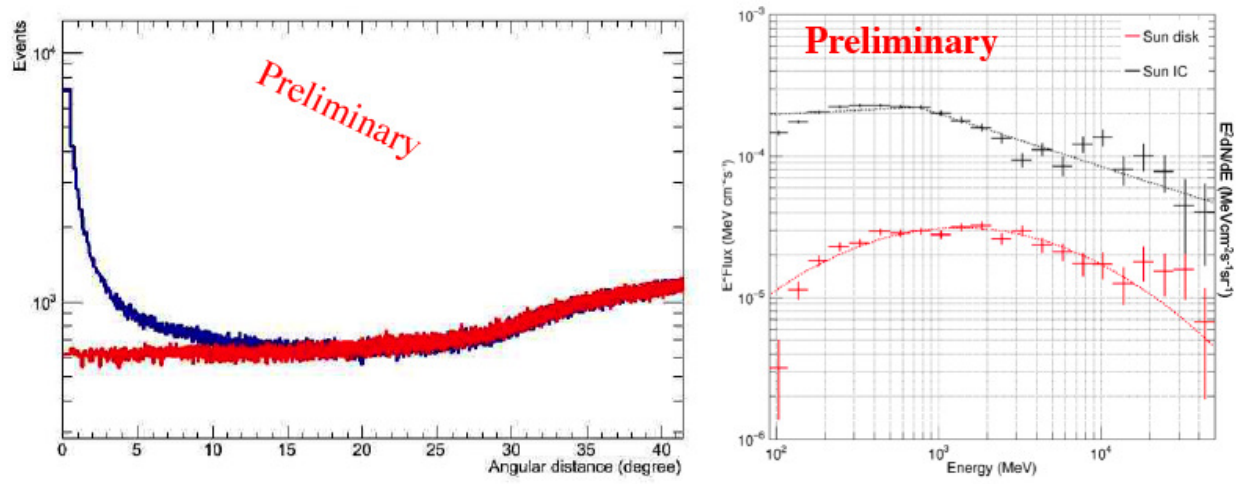

Figure 2. Left: Right: Number of events vs. angular distance from the Sun, for the full data sample (red) and for the off-time source sample (blue). Right: Spectral energy distribution of the two solar components: Sun IC (top, black line), Sun disk (bottom, red line).

Galactic and isotropic (presumably extra-galactic) gamma-ray emission averaged along the ecliptic and to weak point sources. The background is estimated with the trial off-time source method where an off-time source follows the path of the real source but at different times and at $90^{\circ}$ distance (same path at different times). Fig. 2 left shows the distribution of the events for both background and sun samples centered on the solar position, as a function of the angular distance from the fake-sun and the sun respectively. While for the solar centered data set the number of events increases considerably for small elongation angles, the fake-Sun profile is flat. The two distributions overlap at distances larger than $20^{\circ}$ where the signal becomes insignificant.

\section{7-year analysis results}

The full data sample has been modeled fitting the background determined with the off-time source method and masked near the solar disk, with the two emission components from the Sun. The disk emission is modeled as a point-like source with a Log Parabolic energy spectrum, while for IC emission a model undependent approach is used. The extended IC emission is made up of a parametric energy spectrum and a generic $1 / \theta$ radial dependence fitted on data, where $\theta$ is the angular distance from the Sun. Then, the Sun-centered maps are analyzed using a binned maximum likelihood technique developed in Fermi-LAT Science Tools (version v11r03p0). The analysis performed gives a significant detection and separation of the disk and of the IC extended component. Fig.2 right shows the spectral energy distribution (SED) for all solar components evaluated in the first 3 years of FermiLAT data taking. The disk component is well fitted by a Log Parabola with an integral flux above 100 $\mathrm{MeV}$ of $(1.93 \pm 0.07) \times 10^{-7} \mathrm{ph} \mathrm{cm}^{-2} \mathrm{~s}^{-1}$ while we have fitted the IC component with a broken power law resulting in an integral flux above $100 \mathrm{MeV}$ of $(2.02 \pm 0.02) \times 10^{-6} \mathrm{ph} \mathrm{cm}^{-2} \mathrm{~s}^{-1} \mathrm{sr}^{-1}$. As already found in [4] the highest energy points show an excess with respect to model prediction. This result is being carefully investigated on the entire data set period in order to clarify this discrepancy.

\section{Modulation}

Gamma-ray flux measurements depend on the 11-year solar cycle. The maximum in the solar activity corresponds to the minimum CR flux and vice versa. Solar activity reached its maximum in 2014 after 
a period of minimum in the first years of Fermi-LAT data taking. The effect of solar modulation has already been observed analyzing the lunar gamma-ray emission [7]. We are investigating the effects of the solar activity on the fluxes of the two components of the Sun emission. On the basis of the first results, the disk component shows a clear trend in anticorrelation with the solar activity, as expected. The analysis on the IC component is still being investigated but we expect to find a dependence with the solar modulation as well.

\section{Conclusions}

We have analyzed a 7.5-year data sample of Fermi-LAT observations centered on the solar position using the new Pass 8 processing performance. We have used a simplified model that takes into account the background separately evaluated and two emission components from the Sun. The first component from the Sun is a pointlike emission from the disk resulting from the interaction of cosmic rays protons and nuclei with the solar atmosphere. The second contribution comes from the IC scattering of CR electrons and positrons scattering off solar photons in the heliosphere and appears as an extended halo around the Sun. The behavior of the IC spectrum as a function of distance from the Sun is as expected. The solar modulation effect is currently still under investigation, though the first results confirm a clear anticorrelation trend.

\section{Acknowledgements}

The Fermi LAT Collaboration acknowledges generous ongoing support from a number of agencies and institutes that have supported both the development and the operation of the LAT as well as scientific data analysis. These include the National Aeronautics and Space Administration and the Department of Energy in the United States, the Commissariat á l'Energie Atomique and the Centre National de la Recherche Scientifique / Institut National de Physique Nucléaire et de Physique des Particules in France, the Agenzia Spaziale Italiana and the Istituto Nazionale di Fisica Nucleare in Italy, the Ministry of Education, Culture, Sports, Science and Technology (MEXT), High Energy Accelerator Research Organization (KEK) and Japan Aerospace Exploration Agency (JAXA) in Japan, and the K. A. Wallenberg Foundation, the Swedish Research Council and the Swedish National Space Board in Sweden. Additional support for science analysis during the operations phase from the following agencies is also gratefully acknowledged: the Istituto Nazionale di Astrofisica in Italy and the Centre Nationale d'Études Spatiales in France.

\section{References}

[1] Moskalenko, I.V. et al. 2006, ApJL, 652, L65; Orlando, E., \& Strong, A.W., 2006, arXiv:astroph/0607563 (Ap\&SS, 309,359)

[2] Orlando, E. \& Strong A.W., 2008, A\&A, 480, 847

[3] Abdo, A.A., et al., 2010, Physical Review Letters, 104, 101101

[4] Abdo, A.A., et al., 2011, ApJ 734, 116

[5] http : //fermi.gsfc.nasa.gov/ssc/data/analysis/documentation/

Cicerone/Cicerone_LAT_IRF S/IRF_overview.html

[6] The Fermi LAT Collaboration, arXiv:1501.02003, accepted for publication on ApJS

[7] M.Ackermann, et al. Phys. Rev D 93, 082001 (2016) 